\title{
Solar-like stars seismology
}

\section{Dumitru Pricopi and Marian Doru Suran}

\author{
Astronomical Institute of the Romanian Academy, Str. Cutitul de Argint 5, RO-040557 \\ Bucharest, Romania \\ emails: dpricopi@aira.astro.ro, suran@aira.astro.ro
}

\begin{abstract}
Many solar like stars were observed by CoRoT and MOST missions. We try to investigate some of these stars using asteroseismic inversion method using both frequencies fitting and separation fitting. Our results are compared with semiempirical method based on direct power spectrum analysis.
\end{abstract}

Keywords. stars: oscillations, methods: data analysis

\section{Introduction}

In this paper we are focused on the asteroseismic interpretation of the F8V star HD203608. Basic parameters of this star are found using the asteroseismic inversion method i.e. fitting the observed and calculated frequency spectrum. In our study we are using the 21 observed frequencies of this star (Mosser et al. 2008) in a frequency range $[1.5 ; 3.0 \mathrm{mHz}]$. HD203608 is a low-metallicity star of the old galactic disk. The surface metallicity $[\mathrm{Z} / \mathrm{X}]=-0.5 \pm 0.10$ with respect to the solar metallicity (Grevesse \& Noels (1993)). Because this star belongs to the old disk, we shall adopt for the calibration an initial abundance $Y=0.25$. Using photometric data, Thevenin (1998) and more recently Jonsell et al. (2005) and del Peloso et al. (2005) adopted a value of effective temperature: Teff $=6070 \mathrm{~K}$ with an uncertainty of $\pm 100 \mathrm{~K}$. The luminosity were estimeted by Mosser et al. (2008): $L / L_{\odot}=1.39 \pm 0.13$. This star has a low projected rotational velocity: vsini $=2.4 \pm 0.5 \mathrm{~km} / \mathrm{s}$. The age of the star is now determined in the range $7.25 \pm 0.07 \mathrm{Gyr}$.

\section{Results and conclusion}

We computed evolutionary models using CESAM2K stellar evolutionary code for stellar masses between $0.80 \mathrm{M} 8$ and $1.10 \mathrm{M} 8$, with a step of $0.02 \mathrm{M} 8$, for chemical composition $\mathrm{X}=0.71, \mathrm{X}=0.72, \mathrm{Z}=0.006$, a mixing-length parameter of 1.65 times the pressure scale height and no core overshooting. Subsequently, we computed the frequencies of modes having $l=0,1,2,3$ of all the models, using LNAWENR non-adiabatic code. As is wellknown, a necessary condition for a model frequency to be detected at the real star, is that the model frequency to be stable. We tested the stability of model's oscillations using the usual condition $\eta>0$, where $\eta=W / \int_{0}^{1}|d W / d x| d x \propto \operatorname{Im}(\omega) / \operatorname{Re}(\omega), W$ is the total mechanical work done by pulsation, $\omega$ is the dimensionless frequency. The fundamental stellar parameters of the best fitting model for HD203608 are: $M=0.86 M_{\odot}$, Teff $=6100 K, L=1.34 L_{\odot}, R=1.05 R_{\odot}$ and age $=9.35$ Gyr. These values are very similar to those obtained by Mosser et al. (2008). The best fitting model was able to match 5 frequencies in $1 \sigma, 10$ in $2 \sigma$ and 13 in $3 \sigma$. All the calculated frequencies are found to be unstable. Our best fitting model failed to represent the radial modes and non-radial modes with $\mathrm{l}=3$. 


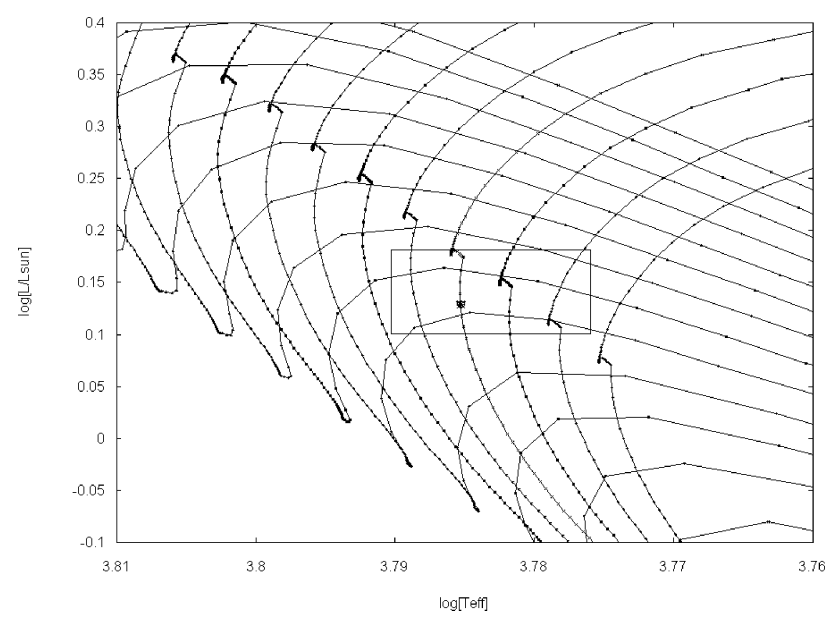

Figure 1. The location of the best fitting model in the grid corresponding to $X=0.72$. This location correspond to a stellar mass of $0.86 M_{\odot}$ and age of $9.35 \mathrm{Gyr}$. The rectangle denotes the error box of observed values of effective temperature and luminosity.

\section{References}

del Peloso, E. F., Cunha, K., da Silva, L., \& Porto de Mello, G. F. 2005, A\& A, 441, 1149

Grevesse, N. \& Noels, A. 1993, Cosmic abundance of the elements, in Origin and evolution of the elements, edited by N. Prantzos, E. Vangioni Flam and M. Casse, Cambridge University, England, pp. 15-25

Jonsell, K., Edvardsson, B., Gustafsson, B. et al. 2005, A\&GA, 440, 231

Mosser, B., Deheuvels, S., Michel, E. et al. 2008, A\&A (to appear)

Thevenin, F. 1998 VisieR Online Data Catalog, 3193, 0 